\title{
Conductance fluctuations, weak localization, and shot noise for a ballistic constriction in a disordered wire
}

\author{
C. W. J. Beenakker and J. A. Melsen \\ Instituut-Lorentz, University of Leiden, P.O. Box 9506, 2300 RA Leiden, The Netherlands
}

(Received 8 March 1994)

\begin{abstract}
This is a study of phase-coherent conduction through a ballistic point contact with disordered leads. The disorder imposes mesoscopic (sample-to-sample) fluctuations and weak-localization corrections on the conductance, and also leads to time-dependent fluctuations (shot noise) of the current. These effects are computed by means of a mapping onto an unconstricted conductor with a renormalized mean free path. The mapping holds in both the metallic and the localized regimes, and permits a solution for an arbitrary ratio of mean free path to sample length. In the case of a single-channel quantum point contact, the mapping is onto a one-dimensional disordered chain, for which the complete distribution of the conductance is known. The theory is supported by numerical
\end{abstract} simulations.

\section{INTRODUCTION}

The problem addressed in this paper is that of phasecoherent electron transport through a ballistic point contact between disordered metals. The geometry is shown schematically in Fig. 1. The same problem was studied recently by Maslov, Barnes, and Kirczenow (MBK), ${ }^{1}$ to whose paper we refer to for an extensive introduction and bibliography. The analytical theory of MBK is limited to the case that the mean free path $l$ for elastic impurity scattering is much greater than the total length $L$ of the system. In this "quasiballistic" case of $l \gg L$, the backscattering through the point contact by disorder in the wide regions can be treated perturbatively. In the present paper we go beyond MBK by solving the problem for arbitrary ratio of $l$ and $L$, from the quasiballistic, through the diffusive, into the localized regime of quantum transport.

Just as in Ref. 1, we model the scattering by the impurities and by the constriction by independent and isotropic transfer matrices. That is to say, we write the transfer matrix $M$ of the whole system as the product $M=M_{2} M_{0} M_{1}$ of the transfer matrices $M_{1}$ and $M_{2}$ of the two wide disordered regions and the transfer matrix $M_{0}$ of the ballistic constriction, and then we as-

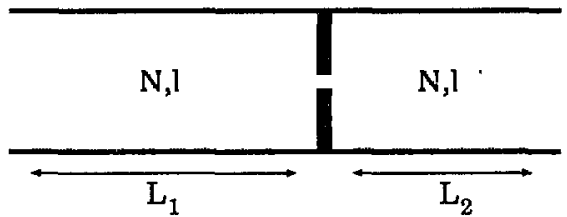

FIG. 1. Schematic illustration of the point-contact geometry, consisting of a ballistic constriction [with conductance $\left.N_{0}\left(2 e^{2} / h\right)\right]$ in a disordered wire (with length $L=L_{1}+L_{2}$, mean free path $l$, and $N$ transverse modes). To define a scattering geometry, the disordered regions (dotted) and the point contact (black) are separated by scattering-free segments. sume that the three transfer matrices are distributed according to independent and isotropic distributions $p_{i}\left(M_{i}\right)$ $(i=0,1,2)$. [A distribution $p(M)$ is called isotropic if it is only a function of the eigenvalues of $M M^{\dagger}$.] The assumption of three independent transfer matrices requires a spatial separation of scattering by the impurities and by the constriction, which prevents us from treating the effects of impurity scattering on the conductance quantization. (This problem has been treated extensively in the past, cf. Ref. 2 for a recent review.) The isotropy assumption for the transfer matrix $M_{0}$ of the constriction is a simple but realistic model of the coupling between wide and narrow regions, which implies that all $N$ transverse modes in the wide regions (of width $W$ ) to the left and right of the constriction (of width $W_{0}$ ) are equally coupled to each other. ${ }^{3}$ The basic requirement here is that the widening from $W_{0}$ to $W$ occurs abruptly and without spatial symmetries. ${ }^{4}$ The isotropy assumption for the transfer matrices $M_{1}$ and $M_{2}$ of the disordered regions (of length $L_{1}$ and $L_{2}$ ) requires aspect ratios $L_{1} / W, L_{2} / W \gg 1$ corresponding to a wire geometry. ${ }^{5}$ Finally, we assume that the impurity scattering is weak in the sense that $l \gg \lambda_{F}$ (with $\lambda_{F}$ the Fermi wavelength). Under these assumptions we can treat the impurity scattering within the framework of the Dorokhov-Mello-Pereyra-Kumar (DMPK) equation. ${ }^{6,7}$

The key result which enables us to go beyond MBK is a mapping between the constricted and unconstricted geometries in Figs. 1 and 2. The unconstricted geometry of Fig. 2 is a disordered wire of length $L=L_{1}+L_{2}$, with $N_{0}$ transverse modes and mean free path $l / \nu$. The number $N_{0}$ is determined by the quantized conductance $N_{0}\left(2 e^{2} / h\right)$ of the point contact in the constricted geometry. The fraction $\nu$ is defined by

$$
\nu=\frac{\beta N_{0}+2-\beta}{\beta N+2-\beta} .
$$

(Here $\beta \in\{1,2,4\}$ is the Dyson index, which equals 1 in zero magnetic field, 2 in a time-reversal-symmetry break- 


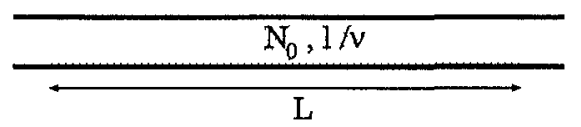

FIG. 2. Unconstricted geometry, with length $L$, mean free path $l / \nu$, and $N_{0}$ transverse modes. The key result of this paper is the equivalence with the constricted geometry of Fig. 1, for $\nu$ given by Eq. (1.1).

ing magnetic field, and 4 in zero field with strong spinorbit scattering.) Starting from the DMPK equation, we will deduce (in Sec. II) that the conductance has the same probability distribution in the two geometries. The equivalence holds for all moments of the conductance, so that it allows us to obtain (in Sec. III) the effect of the point contact on weak localization and universal conductance fluctuations directly from known results for disordered wires ${ }^{8}$ - without the restriction $l \gg L$ of Ref. 1 . It also holds for other transport properties than the conductance (in fact it holds for the complete distribution of the transmission eigenvalues). As an example of current interest, we will compute the suppression of shot noise in the point-contact geometry.

In Sec. IV we consider the case $N_{0}=1$ of a quantum point contact with a single transmitted channel. The mapping is then onto a single-mode wire (or onedimensional chain) of length $L$ and mean free path $\frac{1}{2}(\beta N+2-\beta) l$. The one-dimensional (1D) chain has been studied extensively in the past as the simplest possible system exhibiting localization. ${ }^{9,10}$ The precise correspondence with the problem of a single-channel ballistic constriction in a multichannel disordered wire seems to be both novel and unexpected. From this correspondence we predict that the resistance $R$ of the point contact has an exponential distribution,

$\mathcal{P}(R)=\beta \frac{e^{2}}{h} \frac{N l}{L} \exp \left[-\beta \frac{e^{2}}{h} \frac{N l}{L}\left(R-h / 2 e^{2}\right)\right]$,

$$
R \geq h / 2 e^{2}
$$

provided the disordered wire is metallic $(N l / L \gg 1)$. The width of the distribution decreases by a factor of 2 upon breaking time-reversal symmetry in the absence of spin-orbit scattering $(\beta=1 \rightarrow \beta=2)$.

To test the theoretical predictions we present (in Sec. V) results of numerical simulations, both for $N_{0} \gg 1$ and for $N_{0}=1$. The numerical data for the density of transmission eigenvalues provide independent support for the mapping. In particular, we find good agreement with Eq. (1.2), including the decrease in width upon application of a magnetic field.

\section{MAPPING OF CONSTRICTED ONTO UNCONSTRICTED GEOMETRY}

The first step is to show that the geometry of Fig. 1, with lengths $L_{1}$ and $L_{2}$ of disordered wire to the left and right of the point contact, is equivalent to the geometry of Fig. 3, with a length $L=L_{1}+L_{2}$ of dis-

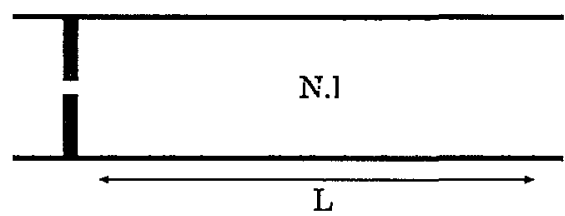

FIG. 3. Constricted geometry with all disorder at one side of the point contact. For isotropic transfer matrices this geometry is statistically equivalent to that of Fig. 1.

ordered wire to one side only. The transfer matrix for Fig. 1 is $M=M_{2} M_{0} M_{1}$, the transfer matrix of Fig. 3 is $M^{\prime}=M_{0} M_{1} M_{2}$. The corresponding probability distributions $p(M)$ and $p^{\prime}\left(M^{\prime}\right)$ are

$$
\begin{aligned}
p & =p_{2} * p_{0} * p_{1}, \\
p^{\prime} & =p_{0} * p_{1} * p_{2},
\end{aligned}
$$

where the symbol $*$ denotes a convolution:

$$
p_{i} * p_{j}(M)=\int d M_{j} p_{i}\left(M M_{j}^{-1}\right) p_{j}\left(M_{j}\right) .
$$

(The invariant measure $d M$ on the group of transfer matrices is introduced in Refs. 7 and 8.) Isotropic distributions have the property that their convolution does not depend on the order: $p_{i} * p_{j}=p_{j} * p_{i}$ if both $p_{i}$ and $p_{j}$ are isotropic (see the Appendix for a proof). It follows that $p=p^{\prime}$, and hence that the geometries of Figs. 1 and 3 are equivalent. Note that the isotropy assumption is crucial here, otherwise the convolution would not commute.

The second step is to show the equivalence of the constricted geometry of Fig. 3 with the unconstricted geometry of Fig. 2. We recall ${ }^{5}$ that the $2 N$ eigenvalues of the transfer matrix product $M M^{\dagger}$ come in inverse pairs $\exp \left( \pm \mathbf{2} x_{n}\right), n=1,2, \ldots, N$. The ratio $L / x_{n} \in[0, \infty)$ has the significance of a channel-dependent localization length. We define

$$
\begin{aligned}
& T_{n}=1 / \cosh ^{2} x_{n}, \\
& \lambda_{n}=\sinh ^{2} x_{n}=\left(1-T_{n}\right) / T_{n} .
\end{aligned}
$$

The numbers $T_{n} \in[0,1]$ are the transmission eigenvalues (i.e., the eigenvalues of the matrix product $t t^{\dagger}$, with $t$ the $N \times N$ transmission matrix). A ballistic point contact, with conductance $N_{0}\left(2 e^{2} / h\right)$, has to a good approximation $T_{n}=1\left(\lambda_{n}=0\right)$ for $1 \leq n \leq N_{0}$, and $T_{n}=0\left(\lambda_{n} \rightarrow \infty\right)$ for $N_{0}+1 \leq n \leq N$. (This is a statement about transmission eigenvalues, not about the transmission probabilities of individual modes, which are all of order $N_{0} / N$.) The joint probability distribution $P_{N}\left(\lambda_{1}, \lambda_{2}, \ldots, \lambda_{N}, L\right)$ of the $\lambda$ variables depends on the length $L$ of the disordered wire according to the DMPK equation, ${ }^{6,7}$

$$
\frac{1}{2} l(\beta N+2-\beta) \frac{\partial P_{N}}{\partial L}=\sum_{n=1}^{N} \frac{\partial}{\partial \lambda_{n}} \lambda_{n}\left(1+\lambda_{n}\right) J_{N} \frac{\partial}{\partial \lambda_{n}} \frac{P_{N}}{J_{N}},
$$

$$
J_{N}=\prod_{i=1}^{N} \prod_{j=i+1}^{N}\left|\lambda_{i}-\lambda_{j}\right|^{\beta}
$$


In this formulation the ballistic point contact appears as an initial condition

$$
\lim _{L \rightarrow 0} P_{N}=\lim _{\Lambda \rightarrow \infty} \prod_{n=1}^{N_{0}} \delta\left(\lambda_{n}\right) \prod_{n=N_{0}+1}^{N} \delta\left(\lambda_{n}-\Lambda\right) .
$$

The closed channels $N_{0}+1 \leq n \leq N$ are irrelevant for conduction and can be integrated out. The reduced distribution function $\tilde{P}_{N}\left(\lambda_{1}, \lambda_{2}, \ldots, \lambda_{N_{0}}, L\right)$ is defined by

$$
\tilde{P}_{N}=\int_{0}^{\infty} d \lambda_{N_{0}+1} \int_{0}^{\infty} d \lambda_{N_{0}+2} \cdots \int_{0}^{\infty} d \lambda_{N} P_{N}
$$

and satisfies the evolution equation

$$
\begin{aligned}
& \frac{1}{2} l(\beta N+2-\beta) \frac{\partial \tilde{P}_{N}}{\partial L}= \sum_{n=1}^{N_{0}} \frac{\partial}{\partial \lambda_{n}} \lambda_{n}\left(1+\lambda_{n}\right) \\
& \times J_{N_{0}} \frac{\partial}{\partial \lambda_{n}} \frac{\tilde{P}_{N}}{J_{N_{0}}} \\
& \lim _{L \rightarrow 0} \tilde{P}_{N}=\prod_{n=1}^{N_{0}} \delta\left(\lambda_{n}\right) .
\end{aligned}
$$

We now compare with the unconstricted geometry of Fig. 2 , which consists of a wire with $N_{0}$ transverse modes, length $L$, and mean free path $l / \nu$. The probability distribution $P_{N_{0}}\left(\lambda_{1}, \lambda_{2}, \ldots, \lambda_{N_{0}}, L\right)$ for this geometry is determined by

$$
\begin{aligned}
\frac{1}{2}(l / \nu)\left(\beta N_{0}\right. & +2-\beta) \frac{\partial P_{N_{0}}}{\partial L} \\
& =\sum_{n=1}^{N_{0}} \frac{\partial}{\partial \lambda_{n}} \lambda_{n}\left(1+\lambda_{n}\right) J_{N_{0}} \frac{\partial}{\partial \lambda_{n}} \frac{P_{N_{0}}}{J_{N_{0}}},
\end{aligned}
$$

$\lim _{L \rightarrow 0} P_{N_{0}}=\prod_{n=1}^{N_{0}} \delta\left(\lambda_{n}\right)$.

Comparison of Eqs. (2.10) and (2.11) shows that $\tilde{P}_{N}=$ $P_{N_{0}}$ if $\nu$ is given by Eq. (1.1), as advertised in the Introduction.

We will apply the mapping between constricted and unconstricted geometries to study the distribution of transport properties $A$ of the form $A=\sum_{n} a\left(\lambda_{n}\right)$, with $\lim _{\lambda \rightarrow \infty} a(\lambda)=\mathbf{0}$ (so that only the channels $n \leq N_{0}$ contribute). We denote by $\mathcal{P}(A, s)$ and $\mathcal{P}_{0}(A, s)$ the distribution of $A$ in, respectively, the constricted and unconstricted geometries, with $s=L /$ (mean free path). Since the mean free path in the constricted geometry is a factor $\nu$ smaller than in the unconstricted geometry, we conclude that

$$
\mathcal{P}(A, s)=\mathcal{P}_{0}(A, \nu s) .
$$

This is the key result which allows us to solve the problem of a ballistic constriction in a disordered wire, for arbitrary ratio $s$ of wire length to mean free path.

\section{MANY-CHANNEL POINT CONTACT}

In this section we study a point contact which has a conductance much greater than $e^{2} / h$, so that $N_{0} \gg 1$. We mainly consider the metallic regime $N l / L \gg 1$, in which the conductance of the disordered region separately is also much greater than $e^{2} / h$. Two transport properties are studied in detail: First the conductance $G$, given by the Landauer formula

$$
G=G_{0} \sum_{n} T_{n}
$$

where $G_{0}=2 e^{2} / h$ is the conductance quantum; second the shot-noise power $S$, given by ${ }^{11}$

$$
S=S_{0} \sum_{n} T_{n}\left(1-T_{n}\right),
$$

with $S_{0}=2 e|V| G_{0}$ for an applied voltage $V$. We also study the transmission-eigenvalue density, from which other transport properties can be computed. In each case we apply the mapping (2.12) between the constricted and unconstricted geometries. The fraction $\nu$ which rescales the mean free path in this mapping has, according to Eq. (1.1), the series expansion

$$
\nu=\frac{1}{N}\left[N_{0}-(1-2 / \beta)\left(1-N_{0} / N\right)+\mathcal{O}\left(N_{0}^{-1}\right)\right] .
$$

To lowest order, $\nu=N_{0} / N$. The next term, proportional to $1-2 / \beta$, contributes to the weak-localization effect.

\section{A. Weak localization and conductance fluctuations}

The mean $\bar{G}$ and variance $\operatorname{Var} G$ of the conductance distribution $\mathcal{P}_{0}(G, \nu s)$ in the unconstricted geometry were computed by Mello and Stone [Eq. (C23) in Ref. $8]$,

$$
\bar{G} / G_{0}=\frac{N_{0}}{1+\nu s}+\frac{1}{3}(1-2 / \beta)\left(\frac{\nu s}{1+\nu s}\right)^{3}+\mathcal{O}\left(\nu s / N_{0}\right),
$$

$\operatorname{Var} G / G_{0}=\frac{2}{15} \beta^{-1}\left(1-\frac{1+6 \nu s}{(1+\nu s)^{6}}\right)+\mathcal{O}\left(\nu s / N_{0}\right)$.

Substitution of the expansion (3.3) yields for the constricted geometry the average conductance $\bar{G}=G_{\text {series }}+$ $\delta G$, with $G_{\text {series }}$ given by $G_{\text {series }}=G_{0}\left(N_{0}^{-1}+s / N\right)^{-1}$ and $\delta G$ given by (denoting $\gamma \equiv N_{0} s / N$ )

$$
\begin{aligned}
\delta G / G_{0}= & (1-2 / \beta)\left[\frac{1}{3}\left(\frac{\gamma}{1+\gamma}\right)^{3}\right. \\
& \left.+\left(1-\frac{N_{0}}{N}\right) \frac{\gamma}{(1+\gamma)^{2}}\right]+\mathcal{O}(s / N) .
\end{aligned}
$$

The term of order $s / N=L / N l$ can be neglected in 
the metallic regime. The term $G_{\text {series }}$ is the series addition of the Sharvin conductance $G_{\text {Sharvin }}=G_{0} N_{0}$ of the ballistic point contact and the Drude conductance ${ }^{12}$ $G_{\text {Drude }}=G_{0} N l / L$ of the disordered region. The term $\delta G$ is the weak-localization correction to the classical series conductance. This term depends on the ratio $\gamma$ of the Sharvin and Drude conductances as well as on the ratio $N_{0} / N$ of the width of the point contact and the wide regions. In the limit $N_{0} / N \rightarrow 0$ at constant $\gamma$, Eq. (3.6) simplifies to

$$
\delta G / G_{0}=\frac{1}{3}(1-2 / \beta)\left[1-(1+\gamma)^{-3}\right] .
$$

The variance $\operatorname{Var} G$ of the sample-to-sample fluctuations of the conductance around the average depends only on $\gamma$ (to order $s / N$ ). From Eqs. (3.3) and (3.5) we find

$$
\operatorname{Var} G / G_{0}=\frac{2}{15} \beta^{-1}\left(1-\frac{1+6 \gamma}{(1+\gamma)^{6}}\right)
$$

In Fig. 4 we have plotted $\delta G$ and $(\operatorname{Var} G)^{1 / 2}$ as a function of $\gamma=G_{\text {Sharvin }} / G_{\text {Drude. }}$ (The limit $N_{0} / N \rightarrow 0$ is assumed for $\delta G$.) For large $\gamma$ the curves tend to $\delta G_{\infty}=\frac{1}{3}(1-2 / \beta) G_{0}$ and $\operatorname{Var} G_{\infty}=\frac{2}{15} \beta^{-1} G_{0}^{2}$, which are the familiar values ${ }^{8}$ for weak localization and universal conductance fluctuations in a wire geometry without a point contact. These values are universal to the extent that they are independent of wire length and mean free path. The presence of a point contact breaks this universality, but only if the Sharvin conductance is smaller than the Drude conductance. For $\gamma>1$ the universality is quickly restored, according to

$$
\begin{aligned}
\frac{\delta G}{\delta G_{\infty}} & =1-\gamma^{-3}+\mathcal{O}\left(\gamma^{-4}\right), \\
\frac{\operatorname{Var} G}{\operatorname{Var} G_{\infty}} & =1-6 \gamma^{-5}+\mathcal{O}\left(\gamma^{-6}\right) .
\end{aligned}
$$

For $\gamma<1$ both $\delta G$ and $\operatorname{Var} G$ are suppressed by the

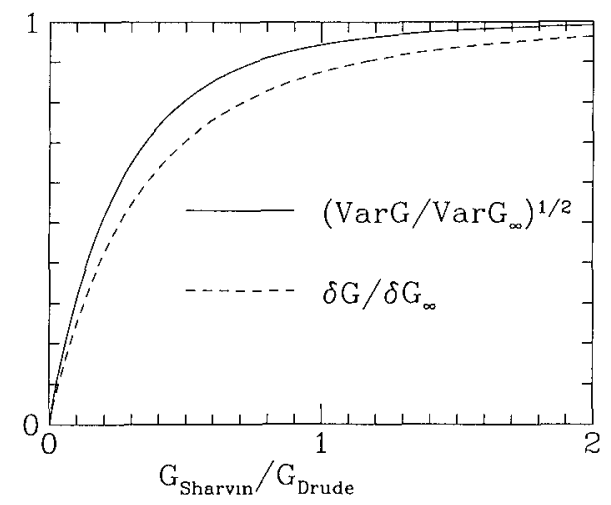

FIG. 4. Suppression by the point contact of the weak-localization correction $\delta G$ and the root-mean-square conductance fluctuations $(\operatorname{Var} G)^{1 / 2}$. The dashed and solid curves are from Eqs. (37) and (3.8), respectively. For $\gamma=G_{\text {Sharvin }} / G_{\text {Drude }}=N_{0} s / N \gg 1$ the curves approach the values $\delta G_{\infty}$ and $\left(\operatorname{Var} G_{\infty}\right)^{1 / 2}$ of an unconstricted disordered wire (normalized to unity in the plot) presence of the point contact, according to

$$
\begin{aligned}
\delta G / G_{0} & =(1-2 / \beta) \gamma+\mathcal{O}\left(\gamma^{2}\right), \\
\operatorname{Var} G / G_{0} & =2 \beta^{-1} \gamma^{2}+\mathcal{O}\left(\gamma^{3}\right) .
\end{aligned}
$$

Maslov, Barnes, and Kirczenow ${ }^{1}$ have studied the quasiballistic regime $l \gg L$. They consider a geometry as in Fig. 1, with $L_{1}=L_{2}$, and relate the variance $\operatorname{Var} G$ of the whole system to the variance $\operatorname{Var} G_{1}$ of one of the two disordered segments of length $\frac{1}{2} L$. Their result (in the present notation) is

$$
\operatorname{Var} G=\gamma^{2}\left(l / L_{1}\right)^{2} \operatorname{Var} G_{1},
$$

in precise agreement with our small- $\gamma$ result (3.12) [since $\operatorname{Var} G_{1}=2 \beta^{-1}\left(L_{1} / l\right)^{2}$ for $\left.l \gg L_{1}\right]$.

So far we have considered the metallic regime $N / s \gg 1$. We now briefly discuss the insulating regime $N / s \ll 1$. In the unconstricted geometry the conductance then has a log-normal distribution, ${ }^{5,13}$

$$
\begin{array}{r}
\mathcal{P}_{0}(G, \nu s)=C \exp \left(-\frac{\left(2 \beta^{-1} \nu s / N_{0}+\ln G / G_{0}\right)^{2}}{8 \beta^{-1} \nu s / N_{0}}\right) \\
\text { if } \nu s / N_{0} \gg 1
\end{array}
$$

with $C$ a normalization constant. The mapping (2.12) implies that the conductance in the constricted geometry has also a log-normal distribution, with mean $\left\langle\ln G / G_{0}\right\rangle=-2 \beta^{-1} s / N$ and variance $\operatorname{Var}\left(\ln G / G_{0}\right)=$ $4 \beta^{-1} s / N$. This distribution is independent of the conductance of the point contact, as long as $N_{0} \gg 1$.

\section{B. Suppression of shot noise}

The average shot-noise power in the unconstricted geometry is [Eq. (A10) in Ref. 14]

$$
\bar{S} / S_{0}=\frac{1}{3} N_{0}(1+\nu s)^{-1}\left[1-(1+\nu s)^{-3}\right]+\mathcal{O}(1) .
$$

The term $\mathcal{O}(1)$ is the weak-localization correction on the shot noise, which is not considered here. The mapping (2.12) implies for the constricted geometry

$$
\bar{S} / S_{0}=\frac{1}{3} N_{0}(1+\gamma)^{-1}\left[1-(1+\gamma)^{-3}\right],
$$

with $\gamma \equiv N_{0} s / N$. Since $S_{0} N_{0}(1+\gamma)^{-1}=2 e|V| G_{\text {series }}=$ $2 e|I|$ (with $I$ the current through the point contact), we can write Eq. (3.16) in terms of the Poisson noise $S_{\text {Poisson }}=2 e|I|$,

$$
\bar{S}=\frac{1}{3} S_{\mathrm{P}_{\text {o1sson }}}\left[1-(1+\gamma)^{-3}\right] .
$$

The suppression of the shot-noise power below the value $S_{\text {Poisson }}$ of a Poisson process is plotted in Fig. 5, as a function of the ratio $\gamma$ of Sharvin and Drude conductances. For $\gamma \ll 1$ the shot noise is zero, as expected for a ballistic constriction. ${ }^{11,15-17}$ For $\gamma \gg 1$ the shot 


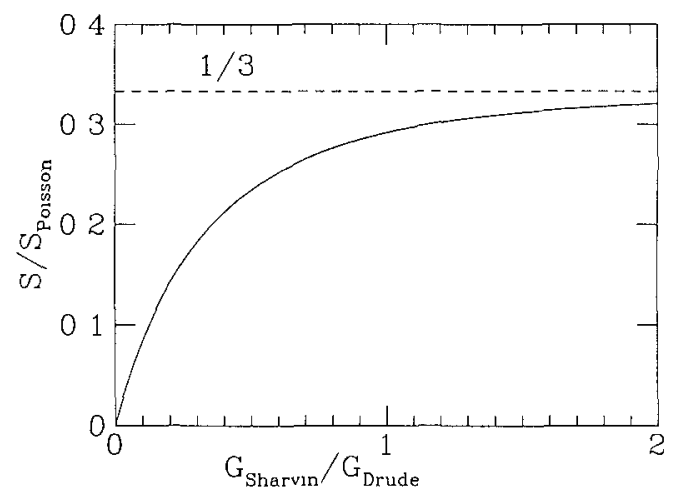

FIG. 5. Suppression of the shot-noise power $S$ below the Poisson noise $S_{\text {Poisson. }}$. The solid curve is computed from Eq. (3.17). The one-third suppression of a diffusive conductor is indicated by the dashed line.

noise is one-third the Poisson noise, as expected for a diffusive conductor. ${ }^{14,18,19}$ The formula (3.17) describes the crossover between these two regimes.

\section{Density of transmission eigenvalues}

We consider the eigenvalue densities

$$
\begin{aligned}
& \rho(x, s)=\left\langle\sum_{n=1}^{N_{0}} \delta\left(x-x_{n}\right)\right\rangle, \\
& \rho(T, s)=\left\langle\sum_{n=1}^{N_{0}} \delta\left(T-T_{n}\right)\right\rangle,
\end{aligned}
$$

which are related by $\rho(T, s)=\rho(x, s)|d T / d x|^{-1}$ (with $\left.T=1 / \cosh ^{2} x\right)$. The (irrelevant) closed channels $n>N_{0}$ have been excluded from the densities. In the unconstricted geometry we have, according to Ref. 20,

$$
\rho(x, \nu s)=\frac{2}{\pi} N_{0} \operatorname{Im} U\left(x-\mathrm{i}^{+}, \nu s\right)+\mathcal{O}(1),
$$

where the complex function $U(z, s)$ is determined by

$U=\operatorname{cotanh}(z-s U), 0>\operatorname{Im}(z-s U)>-\frac{1}{2} \pi$.

The mapping (2.12) implies for the constricted geometry

$$
\rho(x, s)=\frac{2}{\pi} N_{0} \operatorname{Im} U\left(x-\mathrm{i} 0^{+}, N_{0} s / N\right)+\mathcal{O}(1) .
$$

The solution $\rho(x, s)$ of Eqs. (3.21) and (3.22) is plotted in Fig. 6, for several values of $\gamma \equiv N_{0} s / N$. The inset shows the corresponding density of transmission eigenvalues $\rho(T, s)$. For $\gamma \lesssim 1, \rho(T, s)$ has a single peak at unit transmission. For $\gamma \gtrsim 1$ a second peak develops near zero transmission, so that the distribution becomes bimodal. A crossover from unimodal to bimodal distribution on increasing the disorder has also been found in the case of a tunnel barrier. ${ }^{20,21}$ The difference with a point contact is that for a tunnel barrier the single peak is near zero, rather than near unit, transmission.

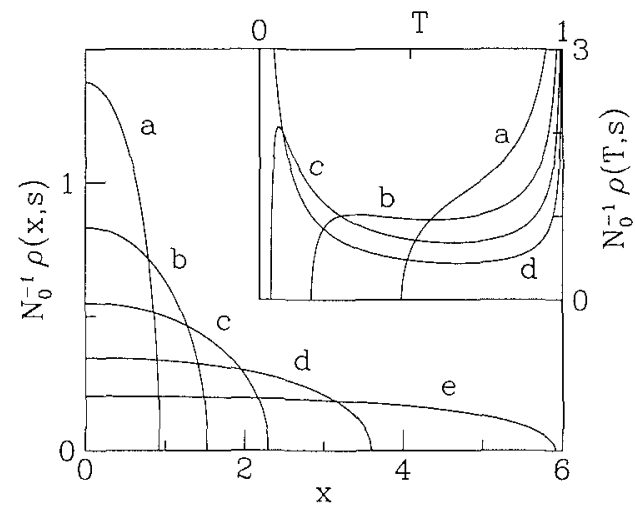

FIG. 6. Density $\rho(x, s)$ as a function of $x$, computed from Eqs. (3.21) and (3.22) for several values of $\gamma \equiv N_{0} s / N$. Curves $a, b, c, d$, and $e$ correspond, respectively, to $\gamma=0.2$, $0.5,1,2$, and 4 . The inset shows the corresponding density $\rho(T, s)=\rho(x, s)|d T / d x|^{-1}$ of transmission eigenvalues $T=1 / \cosh ^{2} x$. Note the crossover from unimodal to bimodal distribution near $\gamma=1$.

\section{SINGLE-CHANNEL POINT CONTACT}

In this section we study a point contact with a quantized conductance of $2 e^{2} / h$, so that $N_{0}=1$. The DMPK equation (2.11) for the distribution $P_{1}\left(\lambda_{1}, L\right) \equiv P(\lambda, L)$ of the single transmitted channel is

$$
\begin{aligned}
& (l / \nu) \frac{\partial}{\partial L} P(\lambda, L)=\frac{\partial}{\partial \lambda} \lambda(1+\lambda) \frac{\partial}{\partial \lambda} P(\lambda, L), \\
& \lim _{L \rightarrow 0} P(\lambda, L)=\delta(\lambda),
\end{aligned}
$$

since $J_{1} \equiv 1$. Equation (1.1) for the fraction $\nu$ which rescales the mean free path becomes

$$
\nu=\frac{2}{\beta N+2-\beta} .
$$

The partial differential equation (4.1) has been studied as early as 1959 in the context of propagation of radio waves through a waveguide with a random refractive index. ${ }^{22,23}$ In the 1980s it was rederived and investigated in great detail, ${ }^{24-28}$ in connection with the problem of localization in a 1D chain. ${ }^{9,10}$ The solution can be written in terms of Legendre functions, or more conveniently in the integral representation

$$
\begin{aligned}
P(\lambda, L)= & (2 \pi)^{-1 / 2}(\nu L / l)^{-3 / 2} e^{-\nu L / 4 l} \\
& \times \int_{\operatorname{arccosh}(1+2 \lambda)}^{\infty} d u \frac{u \exp \left(-u^{2} l / 4 \nu L\right)}{(\cosh u-1-2 \lambda)^{1 / 2}} .
\end{aligned}
$$

According to the Landauer formula (3.1), the conductance $G$ of the whole system is related to the variable $\lambda \equiv(1-T) / T$ by $G=G_{0}(1+\lambda)^{-1}$ (with $G_{0}=2 e^{2} / h$ ). It follows that the resistance $\delta R=1 / G-h / 2 e^{2}$ after subtraction of the contact resistance is just given by $\delta R=\lambda / G_{0}$. In view of the mapping (2.12), the resistance distribution $\mathcal{P}(\delta R, s)$ is given by 


$$
\begin{aligned}
\mathcal{P}(\delta R, s)= & G_{0}(2 \pi)^{-1 / 2}(\nu s)^{-3 / 2} e^{-\nu s / 4} \\
& \times \int_{\operatorname{arccosh}\left(1+2 G_{0} \delta R\right)}^{\infty} d u \frac{u \exp \left(-u^{2} / 4 \nu s\right)}{\left(\cosh u-1-2 G_{0} \delta R\right)^{1 / 2}}
\end{aligned}
$$

The mean and variance of $\delta R$ can be computed either by integrating the distribution (4.4), or directly from the differential equation (4.1). ${ }^{24}$ The result is

$$
\begin{aligned}
\overline{\delta R} & =\frac{1}{2 G_{0}}\left(e^{2 \nu s}-1\right) \\
\operatorname{Var} \delta R & =\frac{1}{6 G_{0}^{2}}\left(e^{6 \nu s}-\frac{3}{2} e^{4 \nu s}+\frac{1}{2}\right) .
\end{aligned}
$$

These results hold in both the metallic and the insulating regimes. We now consider in some more detail the metallic regime $N / s \gg 1$. This implies $\nu s \ll 1$. Equations (4.5) and (4.6) reduce to

$$
\overline{\delta R}=\frac{2 s}{G_{0}}(\beta N+2-\beta)^{-1}+\mathcal{O}(s / N)^{2}=(\operatorname{Var} \delta R)^{1 / 2} .
$$

The complete distribution of the resistance $\delta R$ [which follows from Eq. (4.4) in the limit $\nu s \ll 1]$ is the exponential distribution

$$
\mathcal{P}(\delta R, s)=\frac{G_{0}}{\nu s} \exp \left(-\frac{G_{0}}{\nu s} \delta R\right), \delta R \geq 0 .
$$

For $N \gg 1$ the width $\nu s \simeq 2 s / \beta N$ of the distribution (4.8) has the $1 / \beta$ dependence announced in the Introduction [Eq. (1.2)]. In Fig. 7 we have plotted the exact distribution (4.4) (solid curves) for several values of $s$ and compared with the metallic limit (4.8) (dashed curves). For $\nu s \lesssim 0.1$ (curves labeled $a$ ) the two results are almost indistinguishable.

To make connection with some of the recent literature,

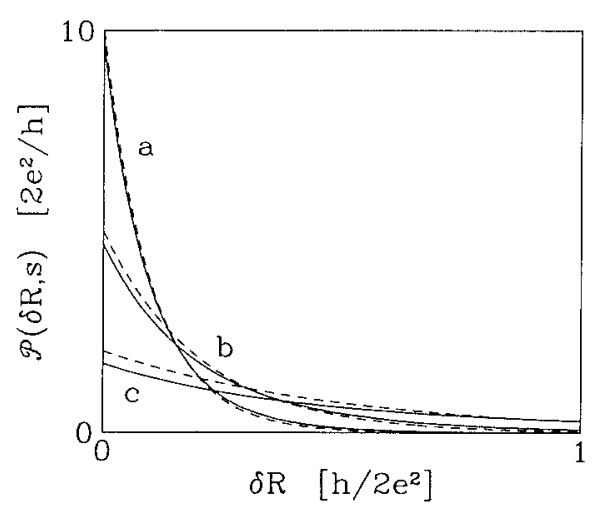

FIG. 7. Probability distribution of the resistance $\delta R=R-h / 2 e^{2}$ of a single-channel point contact, for several values of $\nu s=2(L / l)(\beta N+2-\beta)^{-1}$. Curves $a, b$, and $c$ correspond, respectively, to $\nu s=0.1,0.2$, and 0.5 . The solid curves are computed from Eq. (4.4), the dashed curves are the exponential distribution (4.8) which is approached in the metallic regime $\nu s \ll 1$. we remark that the exponential resistance distribution (4.8) implies for the conductance the distribution

$$
\mathcal{P}(G, s)=\frac{G_{0}}{\nu s} G^{-2} \exp \left(\frac{1-G_{0} / G}{\nu s}\right), 0 \leq G \leq G_{0}
$$

which is strongly peaked at $G=G_{0}$. This is completely different from the conductance distribution of a quantum dot which is weakly coupled by two point contacts to electron reservoirs. ${ }^{29,30}$

\section{NUMERICAL SIMULATIONS}

To test the analytical predictions we have carried out numerical simulations of the Anderson model in the geometry of Fig. 3, using the recursive Green's function technique. ${ }^{31}$ The disordered region (dotted) was modeled by a tight-binding Hamiltonian on a square lattice (lattice constant $a$ ), with a random impurity potential at each site (uniformly distributed between $\pm \frac{1}{2} U_{d}$ ). The constriction was introduced by assigning a large potential energy to sites at one end of the lattice (black in Fig. 1), so as to create a nearly impenetrable barrier with an opening in the center. The constriction itself contained no disorder (the disordered region started at two sites from the barrier). The Fermi energy was chosen at $E_{F}=1.5 u_{0}$ from the band bottom (with $u_{0} \equiv \hbar^{2} / 2 m a^{2}$ ). The ratio $s$ of sample length to mean free path which appears in the theory was computed numerically from $\operatorname{Tr} t_{d} t_{d}^{\dagger}=N(1+s)^{-1}$, with $t_{d}$ the transmission matrix of the disordered region without the constriction. ${ }^{32}$

The simulations for the many-channel and singlechannel point contact are discussed in two separate subsections.

\section{A. Many-channel point contact}

Two geometries were considered for the wide disordered region: a square geometry $(L=W=285 a$, corresponding to $N=119$ ), and a rectangular geometry ( $L=285 a, W=93 a$, corresponding to $N=39$ ). In each case the width of the constriction was $\frac{1}{3} W$ (corresponding to $N_{0}=40$ and $N_{0}=13$ in the square and rectangular geometries, respectively). The length of the constriction was one site. The strength $U_{d}$ of the impurity potential was varied between 0 and $1.5 u_{0}$, corresponding to $s$ between 0 and 11.7.

In Fig. 8 we compare the integrated eigenvalue density $N_{0}^{-1} \int_{0}^{x} d x^{\prime} \rho\left(x^{\prime}, s\right)$, which is the quantity following directly from the simulation. The points are raw data from a single sample. (Sample-to-sample fluctuations are small, because the $x_{n}$ 's are self-averaging quantities. ${ }^{5}$ ) The data are in good agreement with the analytical result of Sec. IIIC, without any adjustable parameters. No significant geometry dependence was found (compare open and closed symbols in Fig. 8). 


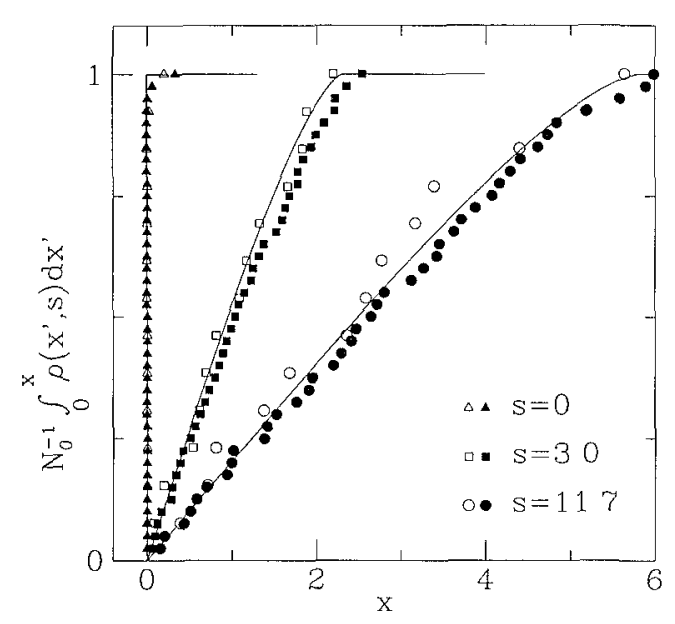

FIG. 8. Comparison between theory and simulation of the integrated eigenvalue density for $N_{0} / N=1 / 3$ and for three different disorder strengths $(s=0,3,11.7)$. Solid curves are from Eqs. (3.21) and (3.22), data points are the $N_{0}$ smallest $x_{n}$ 's from the simulation plotted in ascending order versus $n / N_{0}$ [filled data points are for a square geometry, open points for a rectangular disordered region $(L / W=3)]$.

\section{B. Single-channel point contact}

We considered a square geometry $(L=W=47 a$, corresponding to $N=20$ ), and a rectangular geometry $(L=47 a, W=23 a$, corresponding to $N=10)$. The point contact was three sites wide and two sites long, corresponding to $N_{0}=1$. (The conductance in the absence of disorder was within $5 \%$ of $2 e^{2} / h$.) The distribution $P(\delta R, s)$ of the resistance $\delta R \equiv R-h / 2 e^{2}$ was computed by collecting data for some $10^{4}$ realizations of the impurity potential. To compare the cases $\beta=1$ and $\beta=2$, we repeated the simulations in the presence of a magnetic field of 50 flux quanta $h / e$ through the disordered region. (The magnetic field was graded to zero in the ideal leads.) Two disorder strengths were considered: $U_{d}=1.5 u_{0}$ (corresponding to $s=1.8$ ) and $U_{d}=3.0 u_{0}$ (corresponding to $s=8.3$ ). The results are collected in Fig. 9 and are in good agreement with the theoretical prediction (4.4), again without any adjustable parameters. The theory agrees comparably well with the simulations for the square and rectangular geometries, which shows that the condition $L \gg W$ for the validity of the DMPK equation can be relaxed to a considerable extent.

We find it altogether quite remarkable that the amusingly simple mapping (2.12) between the constricted and unconstricted geometries is capable of reliably predicting the complete distribution of the point-contact resistance, including the effect of broken time-reversal symmetry. We know of no other conventional theoretical technique which could do the same.

\section{ACKNOWLEDGMENTS}

Valuable discussions with M. J. M. de Jong, D. L. Maslov, and B. Rejaei are gratefully acknowledged. This
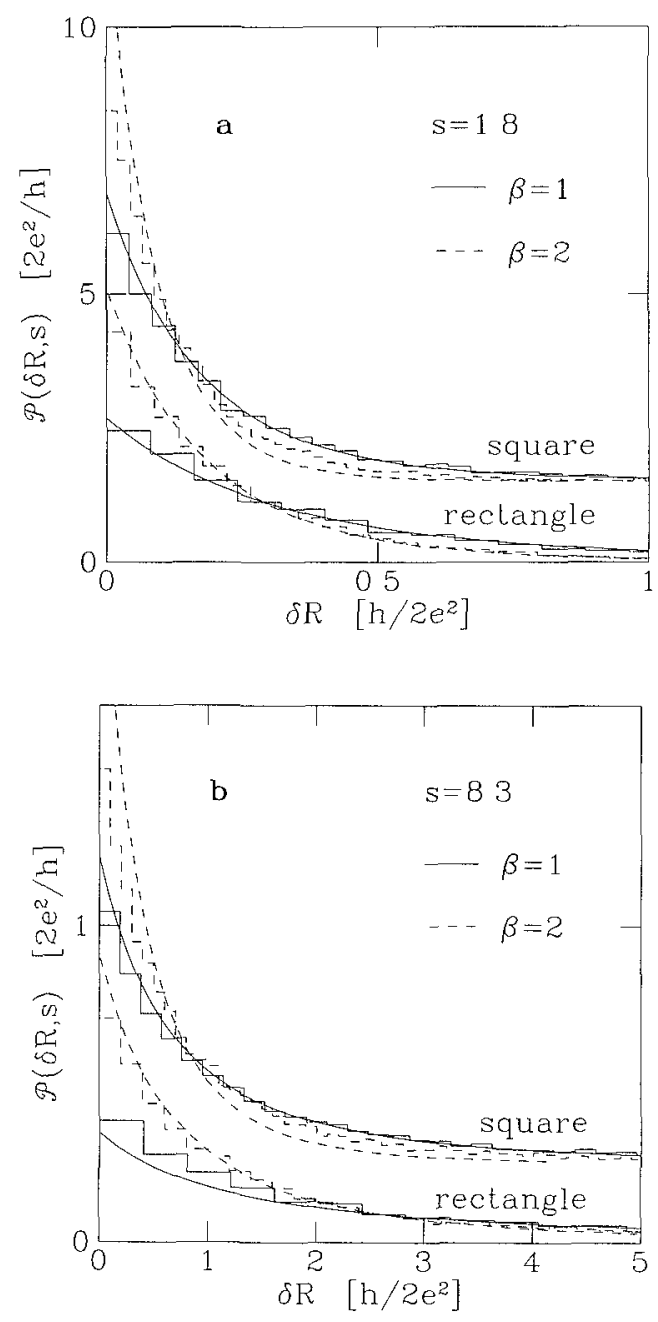

FIG. 9. Comparison between theory and simulation of the distribution of the excess resistance $\delta R$ of a single-channel point contact, for $s=1.8$ (a) and $s=8.3(\mathrm{~b})$. The histograms are the numerical data (for square and rectangular disordered regions), the smooth curves are computed from Eq. (4.4) without any adjustable parameters. Solid curves are for zero magnetic field $(\beta=1)$, dash-dotted curves for a magnetic flux of $50 \mathrm{~h} / \mathrm{e}$ through the disordered region $(\beta=2)$. For clarity, the curves for the square geometry are offset vertically by 1.5 and 0.25 in (a) and (b), respectively.

research was supported by the "Nederlandse organisatie voor Wetenschappelijk Onderzoek" (NWO) and by the "Stichting voor Fundamenteel Onderzoek der Materie" (FOM).

\section{APPENDIX: ISOTROPICALLY DISTRIBUTED TRANSFER MATRICES COMMUTE}

We wish to show that the probability distribution $p(M)$ of a product $M=M_{1} M_{2} M_{3} \cdots$ of transfer matrices is independent of the order of the matrix product, under the assumption that the matrices $M_{\imath}$ are independently distributed with isotropic distributions $p_{2}$. Since $p=p_{1} * p_{2} * p_{3} \cdots$, it is sufficient to show that the convolution 


$$
p_{i} * p_{j}(M)=\int d M_{j} p_{i}\left(M M_{j}^{-1}\right) p_{j}\left(M_{j}\right)
$$

of any two isotropic distributions commutes.

By definition, the distribution $p(M)$ is isotropic if it is only a function of the eigenvalues of the product $M M^{\dagger}$. This implies that $p(M)=p\left(M^{T}\right)$. (The superscripts $\dagger$ and $T$ denote, respectively, the Hermitian conjugate and the transpose of a matrix.) As shown by Mello and coworkers ${ }^{7,8}$ the convolution of two isotropic distributions is again isotropic. Hence

$$
\begin{aligned}
p_{i} * p_{j}(M) & =p_{i} * p_{j}\left(M^{T}\right) \\
& =\int d M_{j} p_{i}\left(M^{T} M_{j}^{-1}\right) p_{j}\left(M_{j}\right) \\
& =\int d M_{j} p_{i}\left(M_{j}^{T^{-1}} M\right) p_{j}\left(M_{j}^{T}\right) \\
& =\int d M_{i} p_{i}\left(M_{i}\right) p_{j}\left(M M_{i}^{-1}\right)=p_{j} * p_{i}(M),
\end{aligned}
$$

which proves the commutativity of the convolution of isotropic distributions.
${ }^{1}$ D. L. Maslov, C. Barnes, and G. Kirczenow, Phys. Rev. Lett. 70, 1984 (1993); Phys. Rev. B 48, 2543 (1993).

${ }^{2}$ S. Das Sarma and S. He, Int. J. Mod. Phys. 7, 3375 (1993).

${ }^{3}$ A. Szafer and A. D. Stone, Phys. Rev. Lett. 62, 300 (1989).

${ }^{4}$ It was assumed in Ref. 1 that the constriction has a spatial symmetry such that coupling between even and odd modes is forbidden in the absence of disorder. This assumption seems to be irrelevant in the presence of disorder.

${ }^{5}$ A. D. Stone, P. A. Mello, K. A. Muttalib, and J.-L. Pichard, in Mesoscopic Phenomena in Solids, edited by B. L. Al'tshuler, P. A. Lee, and R. A. Webb (North-Holland, Amsterdam, 1991).

${ }^{6}$ O. N. Dorokhov, Pis'ma Zh. Eksp. Teor. Fiz. 36, 259 (1982) [JETP Lett. 36, 318 (1982)].

${ }^{7}$ P. A. Mello, P. Pereyra, and N. Kumar, Ann. Phys. (N.Y.) 181, 290 (1988).

${ }^{8}$ P. A. Mello and A. D. Stone, Phys. Rev. B 44, 3559 (1991).

${ }^{9}$ R. Landauer, Philos. Mag. 21, 863 (1970).

${ }^{10}$ P. W. Anderson, D. J. Thouless, E. Abrahams, and D. S. Fisher, Phys. Rev. B 22, 3519 (1980).

${ }^{11}$ M. Büttiker, Phys. Rev. Lett. 65, 2901 (1990).

${ }^{12}$ The Drude formula for the conductance is $G_{\text {Drude }}=$ $\alpha_{d} G_{0} N l_{\mathrm{tr}} / L$, with $l_{\mathrm{tr}}$ the transport mean free path and $\alpha_{d}$ a number which depends on the dimensionality $d$ of the density of states: $\alpha_{2}=\pi / 2$ (Fermi circle) and $\alpha_{3}=4 / 3$ (Fermi sphere). A 1D chain has $\alpha_{1}=2$. These numerical coefficients are absorbed into the mean free path $l \equiv \alpha_{d} l_{\text {tr }}$ which appears in the DMPK equation.

${ }^{13}$ J.-L. Pichard, in Quantum Coherence in Mesoscopic Systems, Vol. 254 of NATO Advanced Study Institute, Series B: Physics, edited by B. Kramer (Plenum, New York, 1991).

${ }^{14}$ M. J. M. de Jong and C. W. J. Beenakker, Phys. Rev. B 46, 13400 (1992).

${ }^{15}$ I. O. Kulik and A. N. Omel'yanchuk, Fiz. Nizk. Temp. 10, 305 (1984) [Sov. J. Low Temp. Phys. 10, 158 (1984)].

${ }^{16}$ V. A. Khlus, Zh. Eksp. Teor. Fiz. 93, 2179 (1987) [Sov.
Phys. JETP 66, 1243 (1987)].

${ }^{17}$ G. B. Lesovik, Pis'ma Zh. Eksp. Teor. Fiz. 49, 513 (1989) [JETP Lett. 49, 592 (1989)].

${ }^{18}$ C. W. J. Beenakker and M. Büttiker, Phys. Rev. B 46, 1889 (1.992).

${ }^{19}$ K. E. Nagaev, Phys. Lett. A 169, 103 (1992).

${ }^{20}$ C. W. J. Beenakker, B. Rejaei, and J. A. Melsen, Phys. Rev. Lett. 72, 2470 (1994).

${ }^{21}$ Yu. V. Nazarov (unpublished).

${ }^{22}$ M. E. Gertsenshtein and V. B. Vasil'ev, Teor. Veroyatn. Primen. 4, 424 (1959) [Theor. Probab. Appl. 4, 391 (1959); 5, 3(E) (1960) [5, 340(E) (1960)]; Radiotekhn. Elektr. 4, 611 (1959) [Radio Eng. Electr. 4, 75 (1959)].

${ }^{23}$ G. C. Papanicolaou, SIAM J. Appl. Math. 21, 13 (1971).

${ }^{24}$ V. I. Mel'nikov, Fiz. Tverd. Tela (Leningrad) 23, 782 (1981) [Sov. Phys. Solid State 23, 444 (1981)].

${ }^{25}$ A. A. Abrikosov, Solid State Commun. 37, 997 (1981).

${ }^{26}$ P. D. Kirkman and J. B. Pendry, J. Phys. C 17, 5707 (1984).

${ }^{27}$ N. Kumar, Phys. Rev. B 31, 5513 (1985).

${ }^{28}$ P. A. Mello, J. Math. Phys. 27, 2876 (1986).

${ }^{29}$ V. N. Prigodin, K. B. Efetov, and S. Iida, Phys. Rev. Lett. 71, 1230 (1993); P. W. Brouwer and C. W. J. Beenakker (unpublished).

${ }^{30}$ R. A. Jalabert, J.-L. Pichard, and C. W. J. Beenakker, Europhys. Lett. (to be published); H. U. Baranger and P. A. Mello (unpublished).

${ }^{31}$ H. U. Baranger, D. P. DiVincenzo, R. A. Jalabert, and A. D. Stone, Phys. Rev. B 44, 10637 (1991). The computer code for the recursive Green's function calculation was kindly made available to us by Dr. Jalabert.

${ }^{32}$ The identification $\operatorname{Tr} t_{d} t_{d}^{\dagger}=N(1+s)^{-1}$ has the status of an interpolation formula between the Sharvin and Drude conductances, which differs by only a few percent from the exact result: M. J. M. de Jong, Phys. Rev. B 49, 7778 (1994). 


$$
p_{i} * p_{j}(M)=\int d M_{j} p_{i}\left(M M_{j}^{-1}\right) p_{j}\left(M_{j}\right)
$$

of any two isotropic distributions commutes.

By definition, the distribution $p(M)$ is isotropic if it is only a function of the eigenvalues of the product $M M^{\dagger}$. This implies that $p(M)=p\left(M^{T}\right)$. (The superscripts $\dagger$ and $T$ denote, respectively, the Hermitian conjugate and the transpose of a matrix.) As shown by Mello and coworkers, ${ }^{7,8}$ the convolution of two isotropic distributions is again isotropic. Hence

$$
\begin{aligned}
p_{i} * p_{j}(M) & =p_{i} * p_{j}\left(M^{T}\right) \\
& =\int d M_{j} p_{i}\left(M^{T} M_{j}^{-1}\right) p_{j}\left(M_{j}\right) \\
& =\int d M_{j} p_{i}\left(M_{j}^{T^{-1}} M\right) p_{j}\left(M_{j}^{T}\right) \\
& =\int d M_{i} p_{i}\left(M_{i}\right) p_{j}\left(M M_{i}^{-1}\right)=p_{j} * p_{i}(M),
\end{aligned}
$$

which proves the commutativity of the convolution of isotropic distributions.
${ }^{1}$ D. L. Maslov, C. Barnes, and G. Kirczenow, Phys. Rev. Lett. 70, 1984 (1993); Phys. Rev. B 48, 2543 (1993).

${ }^{2}$ S. Das Sarma and S. He, Int. J. Mod. Phys. 7, 3375 (1993).

${ }^{3}$ A. Szafer and A. D. Stone, Phys. Rev. Lett. 62, 300 (1989).

${ }^{4}$ It was assumed in Ref. 1 that the constriction has a spatial symmetry such that coupling between even and odd modes is forbidden in the absence of disorder. This assumption seems to be irrelevant in the presence of disorder.

${ }^{5}$ A. D. Stone, P. A. Mello, K. A. Muttalib, and J.-L. Pichard, in Mesoscopic Phenomena in Solids, edited by B. L. Al'tshuler, P. A. Lee, and R. A. Webb (North-Holland, Amsterdam, 1991).

${ }^{6}$ O. N. Dorokhov, Pis'ma Zh. Eksp. Teor. Fiz. 36, 259 (1982) [JETP Lett. 36, 318 (1982)].

${ }^{7}$ P. A. Mello, P. Pereyra, and N. Kumar, Ann. Phys. (N.Y.) 181, 290 (1988).

${ }^{8}$ P. A. Mello and A. D. Stone, Phys. Rev. B 44, 3559 (1991).

${ }^{9}$ R. Landauer, Philos. Mag. 21, 863 (1970).

${ }^{10}$ P. W. Anderson, D. J. Thouless, E. Abrahams, and D. S. Fisher, Phys. Rev. B 22, 3519 (1980).

${ }^{11}$ M. Büttiker, Phys. Rev. Lett. 65, 2901 (1990).

12 The Drude formula for the conductance is $G_{\text {Drude }}=$ $\alpha_{d} G_{0} N l_{\mathrm{tr}} / L$, with $l_{\mathrm{tr}}$ the transport mean free path and $\alpha_{d}$ a number which depends on the dimensionality $d$ of the density of states: $\alpha_{2}=\pi / 2$ (Fermi circle) and $\alpha_{3}=4 / 3$ (Fermi sphere). A $1 \mathrm{D}$ chain has $\alpha_{1}=2$. These numerical coefficients are absorbed into the mean free path $l \equiv \alpha_{d} l_{\mathrm{tr}}$ which appears in the DMPK equation.

13 J.-L. Pichard, in Quantum Coherence in Mesoscopic Systems, Vol. 254 of NATO Advanced Study Institute, Series B: Physics, edited by B. Kramer (Plenum, New York, 1991).

${ }^{14}$ M. J. M. de Jong and C. W. J. Beenakker, Phys. Rev. B 46, 13400 (1992).

${ }^{15}$ I. O. Kulik and A. N. Omel'yanchuk, Fiz. Nizk. Temp. 10, 305 (1984) [Sov. J. Low Temp. Phys. 10, 158 (1984)].

${ }^{16}$ V. A. Khlus, Zh. Eksp. Teor. Fiz. 93, 2179 (1987) [Sov.
Phys. JETP 66, 1243 (1987)].

${ }^{17}$ G. B. Lesovik, Pis'ma Zh. Eksp. Teor. Fiz. 49, 513 (1989) [JETP Lett. 49, 592 (1989)].

${ }^{18}$ C. W. J. Beenakker and M. Büttiker, Phys. Rev. B 46, 1889 (1992).

${ }^{19}$ K. E. Nagaev, Phys. Lett. A 169, 103 (1992).

${ }^{20}$ C. W. J. Beenakker, B. Rejaei, and J. A. Melsen, Phys. Rev. Lett. 72, 2470 (1994).

${ }^{21} \mathrm{Yu}$. V. Nazarov (unpublished).

${ }^{22}$ M. E. Gertsenshtein and V. B. Vasil'ev, Teor. Veroyatn. Primen. 4, 424 (1959) [Theor. Probab. Appl. 4, 391 (1959); 5, 3(E) (1960) [5, 340(E) (1960)]; Radiotekhn. Elektr. 4, 611 (1959) [Radio Eng. Electr. 4, 75 (1959)].

${ }^{23}$ G. C. Papanicolaou, SIAM J. Appl. Math. 21, 13 (1971).

${ }^{24}$ V. I. Mel'nikov, Fiz. Tverd. Tela (Leningrad) 23, 782 (1981) [Sov. Phys. Solid State 23, 444 (1981)].

25 A. A. Abrikosov, Solid State Commun. 37, 997 (1981).

${ }^{26}$ P. D. Kirkman and J. B. Pendry, J. Phys. C 17, 5707 (1984).

${ }^{27}$ N. Kumar, Phys. Rev. B 31, 5513 (1985).

${ }^{28}$ P. A. Mello, J. Math. Phys. 27, 2876 (1986).

${ }^{29}$ V. N. Prigodin, K. B. Efetov, and S. Iida, Phys. Rev. Lett. 71, 1230 (1993); P. W. Brouwer and C. W. J. Beenakker (unpublished).

${ }^{30}$ R. A. Jalabert, J.-L. Pichard, and C. W. J. Beenakker, Europhys. Lett. (to be published); H. U. Baranger and P. A. Mello (unpublished).

${ }^{31}$ H. U. Baranger, D. P. DiVincenzo, R. A. Jalabert, and A. D. Stone, Phys. Rev. B 44, 10637 (1991). The computer code for the recursive Green's function calculation was kindly made available to us by Dr. Jalabert.

32 The identification $\operatorname{Tr} t_{d} t_{d}^{\dagger}=N(1+s)^{-1}$ has the status of an interpolation formula between the Sharvin and Drude conductances, which differs by only a few percent from the exact result: M. J. M. de Jong, Phys. Rev. B 49, 7778 (1994) 